\title{
SARS-CoV-2 and the next generations: which impact on reproductive tissues?
}

\author{
Luisa Zupin $^{1}$ (D) $\cdot$ Lorella Pascolo $^{1} \cdot$ Gabriella Zito $^{1} \cdot$ Giuseppe Ricci $^{1,2} \cdot$ Sergio Crovella ${ }^{1,2}$
}

Received: 4 June 2020 / Accepted: 3 August 2020 / Published online: 11 August 2020

(C) Springer Science+Business Media, LLC, part of Springer Nature 2020

\begin{abstract}
Coronavirus disease 2019 (COVID-19) caused by the severe acute respiratory syndrome-coronavirus 2 (SARS-CoV-2) is a severe global pandemic, affecting mostly the respiratory system. Understandably, attention is also being directed towards the urogenital tract. In this work, expression patterns of various host molecules possibly involved in viral entry and replication were investigated in human female and male reproductive systems by inquiring online repositories, including the Human Protein Atlas, GTEx, FANTOM5. Our findings highlight that male reproductive tissues could be targeted by SARS-CoV-2, particularly the testis since it co-expresses the receptor (ACE2) and the protease (TMPRSS) needed for viral entry. We hypothesized that SARSCoV-2 infection could have repercussions on the fertility status of male individuals Potential infectivity of SARS-CoV-2 in reproductive tissues should be considered in reproductive medicine and management of in vitro fertilization in present and future generations.
\end{abstract}

Keywords Gene expression $\cdot$ SARS-CoV-2 $\cdot$ Reproductive tissues

Coronavirus disease 2019 (COVID-19) caused by the severe acute respiratory syndrome-coronavirus 2 (SARS-CoV-2) is widely considered as a dramatic global pandemic.

COVID-19 has been firstly characterized by respiratory manifestations ranging from sore throat, rhinorrhea, cough, and dyspnea, to pneumonia and acute respiratory distress syndrome with pathognomonic chest X-ray features [1]. Other not specific symptoms are fever, myalgia, headache, lymphopenia, increment of C-reactive protein, hyposmia, and hypogeusia [1]. Nevertheless, new insights highlight that other organs can be infected by SARS-CoV-2; indeed, in a minor fraction of the cases, diarrhea, nausea, and vomiting are reported, suggesting the involvement of the gastro-enteric system [1]. Furthermore,

Electronic supplementary material The online version of this article (https://doi.org/10.1007/s10815-020-01917-0) contains supplementary material, which is available to authorized users.

Luisa Zupin

luisa.zupin@burlo.trieste.it

1 Institute for Maternal and Child Health, IRCCS Burlo Garofolo, Via dell'Istria 65/1, 34137 Trieste, Italy

2 Department of Medicine, Surgery and Health Sciences, University of Trieste, Trieste, Italy proteinuria and acute renal tubular damage in COVID-19 patients indicate kidney impairment [2] and elevated troponin $\mathrm{T}$ and $\mathrm{N}$-terminal pro B-type natriuretic peptide levels underline possible cardiovascular injury [3].

The scientific community is currently focused on the study of SARS-CoV-2 virus characteristics and on the mechanisms leading to the development of COVID-19 disease. Moreover, intense efforts are being made to discover possible treatments/vaccines. Therefore, to date, few studies investigated the possible impact of SARS-CoV-2 infection and sequelae in male and female reproductive systems $[4,5]$, as well as the potential outcome in the context of reproductive medicine.

Focusing on the human reproductive system, we firstly reviewed the scientific literature looking for the main proteins (as well as mRNA expression) already known to be involved in virus entry/replication, whose involvement has been demonstrated through in vitro experiments. Angiotensinconverting enzyme 2 (ACE2) is widely recognized as the primary receptor for virus entry [6,7], and notably, both ACE2 and renin-angiotensin system (RAS) are known to play fundamental roles in human fertility [8]; nevertheless, other actors should be necessary to trigger SARS-CoV-2 virulence. SARS-CoV-2 necessitates of protein spike (S) priming to allow the fusion of viral envelope with the cellular membrane and this process is mediated by host protease such as the type 
II membrane serine proteases (TMPRSS) 2, 4, 11A, 11D, and $11 \mathrm{E}[6,7]$. Moreover, phosphatidylinositol 3-phosphate 5-kinase (PIKFYVE) involved in the endosome dynamics, two pore channel subtype 2 (TPCN2), cathepsin L (CTSL) and cathepsin $\mathrm{B}$ (CTSB) acting in lysosome have been proposed as required molecules for SARS-CoV-2 entry $[6,7]$.

Aimed at investigating the expression pattern of the abovementioned molecules in the female and male reproductive tissues, a databank query and interrogation of the human protein atlas database which reports both the protein production and the gene expression profile (www.proteinatlas.org) were performed; the Genotype-Tissue Expression (GTEx) (www. gtexportal.org) and the Functional Annotation of Mammalian Genomes 5 (FANTOM5) (https://fantom.gsc.riken.jp/5/) repositories, documenting transcriptome findings, were also interrogated. The following molecules, based on previous literature data on SARS-CoV-2 entry mechanisms into host cells, were searched: ACE2; TMPRSS 2, 4, 11A, 11D, and 11E; PIKFYVE; TPCN2; CTSL; CTSB, in the female (vagina, ovary, fallopian tube, endometrium, cervix uterine) and male (ductus deferens, testis, epididymis, seminal vesicle, prostate) reproductive tissues.

Our observations can be accessed in tables 1,2, and 3 of supplementary materials and in Fig. 1.

Interestingly, the protein expression of ACE2 was high in testis (both in cells of the seminiferous duct and Leydig cells) and low in glandular cells of seminal vesicles. Instead, TMPRSS2 has been observed in the epididymis, seminal vesicles, and prostate, and TMPRSS4 has been evidenced in testis, epididymis, and prostate as well as TMPRSS11D has been observed also in seminal vesicles. Therefore, the coexpression of the receptor and almost one protease is present in testis and seminal vesicles, suggesting that they could be potentially infected by SARS-CoV-2.

In the female reproductive system, no tissue showed the presence of ACE2 protein, the same for TMPRSS2, leading us to hypothesize these tissues could not be susceptible to SARS-CoV-2 infection.

Regarding the other factors, no data were available for PIKFYVE; TPCN2 and CTSB were almost produced in all the female and male structures, while CTSL has not been detected or it was present at low level.

Not surprisingly, when looking at the transcriptomic databases (from GTEx, HPA, and FANTOM5), the information reported was different.

$A C E 2$ RNA expression was present in all the reproductive tissues examined, with higher level in testis and lower in the prostate, vagina, fallopian tube, endometrium, and uterine cervix. TMPRSS2 RNA has been also observed in all reproductive tissues but it showed the major expression in the male tissues, namely, ductus deferens, seminal vesicles, and prostate. TMPRSS4, 11A, 11D, and $11 E$ levels were not such conspicuous within the different tissues; nevertheless, the vagina and uterine cervix produced a high amount of them. Finally, the PIKFYVE, TPCN2, CTSL, and CTSB were ubiquitarian in the different reproductive tissues both in males and females.

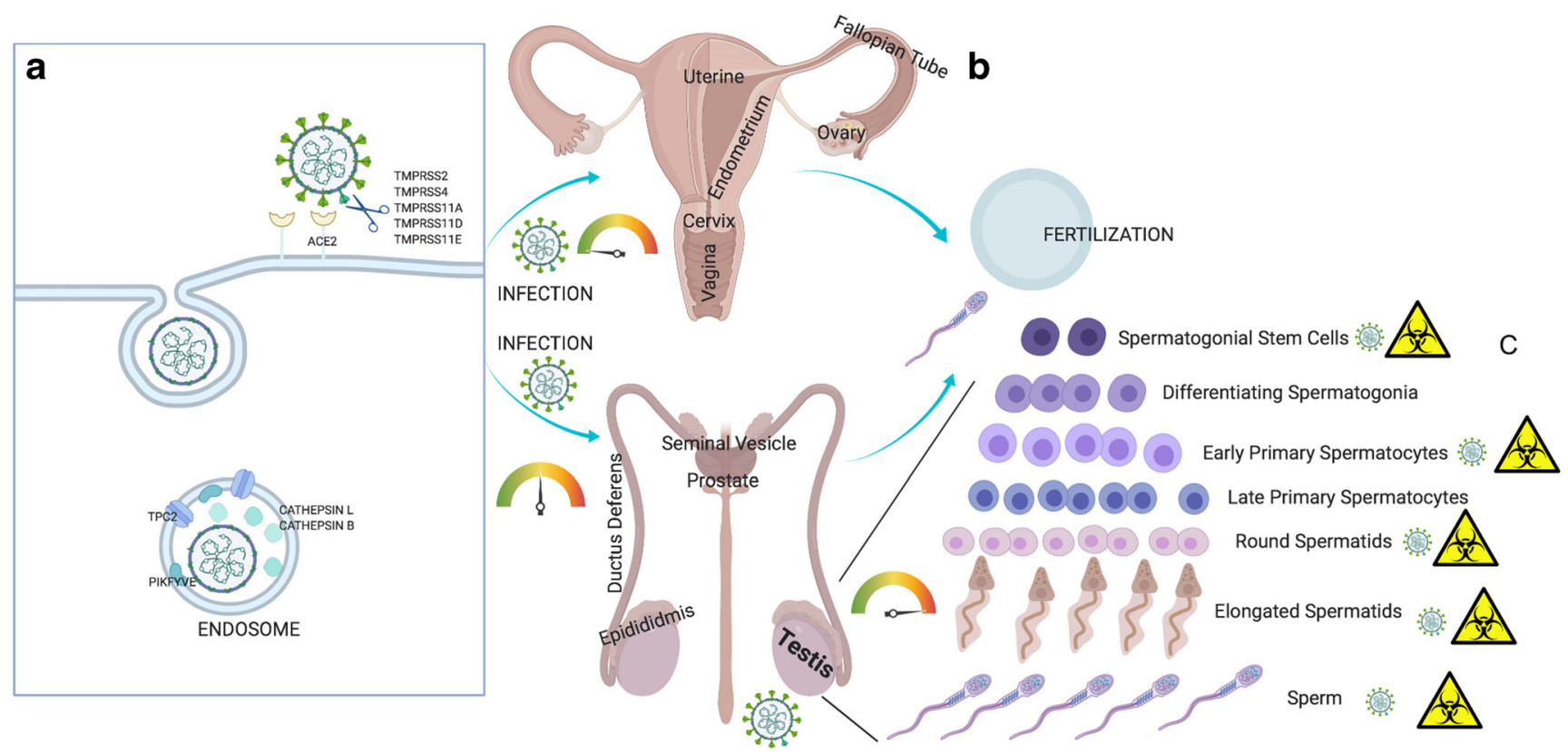

Fig. 1 Schematic representation of the potential SARS-CoV-2 infection in the reproductive system. (A) Viral entry into the cells, highlighting the host factors possibly involved. (B) The potential infectivity of SARS$\mathrm{CoV}-2$ in female (low risk of infection) and male (high risk of infection) reproductive system. (C) The potential infectivity of testis cells based on the co-expression of ACE2 and TMPRSS (the virus image together with hazard icon indicate the cells at the high risk) 
When considering just mRNA expression findings, the data suggest that SARS-CoV-2 could potentially infect all the male and female reproductive tissues.

Special attention should be reserved for the testis, since $A C E 2$ showed in this organ the highest gene expression, taking into consideration that in the lung, the supposed preferential target of SARS-CoV-2, ACE2 presented a lower mRNA expression (consensus among the three dataset: 17.9 and 0.8 normalized expression NX in testis and lung respectively).

It is important to note that the protein data reported based on the search on the human protein atlas are supported by semi-quantitative immunohistochemistry experiments, and not by quantitative proteomics (i.e., western blot or ELISA), so a lack of sensibility towards low amounts of molecules could be hypothesized. Consequently, it is possible to speculate that for this reason a discrepancy between protein and RNA data was observed; post transcriptional changes could also not be excluded.

To further explore this issue, the COVID-19 Cell Atlas was interrogated (https://www.covid19cellatlas.org) looking at the different cell population in the testis, namely differentiating spermatogonia, early primary spermatocytes, elongated spermatids, endothelial cells, late primary spermatocytes, Leydig cells, macrophages, myoid cells, round spermatids, Sertoli cells, sperm, and spermatogonial stem cells. The data of the maximum expression are reported in table 4 of supplementary materials and Fig. 1.

Intriguingly, at low level, ACE2 mRNA expression has been observed in all the cells, specifically, differentiating spermatogonia, early primary spermatocytes, elongated spermatids, endothelial cells, late primary spermatocytes, Leydig cells, macrophages, myoid cells, round spermatids, Sertoli cells, sperm, and spermatogonial stem cells; meanwhile, the TMPRSS $(2,4,11 \mathrm{~A}, 11 \mathrm{D}, 11 \mathrm{E})$ were slightly expressed by elongated spermatids, sperm, and spermatogonial stem cells. Finally, PIKFYVE, TPCN2, CTSL, and CTSB mRNA have been reported in all the different cell types of the testis. These data suggest that spermatids, sperm, spermatogonial stem cells should present a potential risk of susceptibility towards SARS-CoV-2 infection.

The analysis of the testis transcriptome showed that ACE2 positive spermatogonia expressed also an elevated number of genes associated with spermatogenesis respect to ACE2-negative ones; moreover, $A C E 2$-positive Leydig and Sertoli cells (expressing no TMPRSS genes) produced a low amount of mRNA of genes related to mitochondria and reproduction. Therefore, the authors speculated the vulnerability of testis to SARS-CoV-2 infection that could lead to spermatogenesis impairment [9]. Similarly, ACE2 mRNA and protein expression were observed in testis and spermatids $[10,11]$. Moreover, also spermatogonial stem cells, differentiating spermatogonia, leptotene spermatocytes type 1, leptotene spermatocytes type 3, zygotene spermatocytes, diplotene spermatocytes, spermatocyte 7 , spermatids stage 1 , and Sertoli cells may be susceptible for SARS-CoV-2 infection. Testis expressed also TMPRSS2, CTSL, and CSTB, thus possibly corroborating the hypothesis of its vulnerability [12].

Conversely, only a minor fraction of spermatogonial stem cells $(0.05 \%)$ co-expressed ACE2 and TMPRSS2 accordingly with a scRNAseq dataset of human testicular tissue [13].

Regarding SARS-CoV-2 presence in the semen from COVID-19 patients, few studies have been conducted [14]. qRT-PCR of single ejaculated semen sample after a median of 31 days from COVID-19 diagnosis was negative; notably, the $19 \%$ of the infected patients experienced scrotal discomfort, maybe a sign of viral orchitis, although a deep genito-urinary medical examination was not conducted [15]. Similarly, no viral RNA was detected in semen from a patient, after 9 days from the positive naso-pharyngeal swab [16]. Nevertheless, these are preliminary reports, and the second article was conducted only on one patient; moreover, maybe the time of the semen collection after the diagnosis, when the patients were almost recovered front the acute illness, could have affected the negative results. Indeed, SARS-CoV-2 was found in the semen of 6 COVID-19 patients $(15.8 \%), 4$ being in acute stage and 2 in a recovery phase, although the presence of the virus could be due to the failure of the blood barriers around the male reproductive system, it may linger in the apparatus leading to the viremia found in the semen; therefore, the authors warned about the possible sexual transmission of SARS-CoV-2 and the possible preventive measures to be assumed [17].

Our observations obtained using different online tools hint that male reproductive tissues, especially the testis, which coexpresses the receptor and the protease necessary for virus entry could be affected by SARS-CoV-2. To date, few studies were carried out on this topic, and no works investigated the impact of the virus presence on spermatozoa in vitro or in vivo in animal models. Thereafter, the real implication of the infection on the fertility status of the individuals needs to be clarified and proved. It is important to mention that SARS-CoV (outbreak in 2002) promoted orchitis, probably by inducing inflammatory and immunological host response that damages the gonads [18]; considering that ACE2 is the entry receptor of both SARS-CoV and SARS-CoV, it cannot be excluded that the new coronavirus could act in a similar way [1].

Regarding the female reproductive tissues, we observed only low expression of $A C E 2$ in female tissues.

Recently, a co-expression of ACE2 and TMPRSS2 in primate ovarian cells has been observed; meanwhile, in cumulus cells, ACE2 was identified in high amount, but TMPRSS2 was slightly present [13]. In the endometrium, $A C E 2$ expression was reported as low, while the TMPRSS2 one as medium and TMPRSS4, CTSL, and CTSB expressions as high; moreover, all the genes mentioned above showed an increment towards the secretory phase of the menstrual cycle [19]. These findings 
hint a potential although low susceptibility towards SARSCoV-2 infection also in the female reproductive tissues.

On the other hand, the possible risk of the vertical transmission from the mothers to the fetus is under debate. Indeed, SARS-CoV-2-positive newborns are mainly asymptomatic [20], and only some case reports showed the positivity of the immunoglobulins $\operatorname{IgG}$ and $\operatorname{IgM}[21,22]$

The information here reported, being aware that it is based on in silico transcriptomic and proteomic repositories analyses, would be the cue to further deepen SARS-CoV-2 potential impact on the cells from the reproductive tissues; although viral RNA was detected in the semen [17], it is not assessed if the replicative cycle of the virus occurs in the testis or in the related organs, or its presence is only the result of blood barrier failure, even though since SARS-CoV-2 is not quantified in blood [23], the last option can be excluded. Intriguingly, with our observations, we proposed to draw the attention to the coexpression of ACE2 and TMPRSSs in specific cells of the testis, namely, spermatogonial stem cells, spermatids, and spermatozoa, as presumptive targets of SARS-CoV-2. Perhaps, the possible repercussion of SARS-CoV-2 infection on the fertility status of the individuals, especially when COVID-19 occurs in fertile subjects, may be underestimated and should need to be urgently re-evaluated. Physicians and researchers working in the context of reproductive medicine should consider that SARS-CoV-2 is potentially able to infect and possibly replicate in male reproductive tissues with an eventual impact on the infectivity of the semen. Therefore, some questions arise for in vitro fertilization (IVF) technique, about the possibility that spermatozoa could be carriers of the virus and so potentially infect the oocyte during the procedures; consecutively, it is reasonable to ponder about the molecular evaluation of the presence of SARS-CoV-2 in the semen collected in reproductive medicine centers and about the possibility to postpone the IVF procedures, both the gamete preservation and the IVF techniques. Finally, it is important to evaluate the cases of elderly women whose oocyte reserve is diminished, for whom the possible window of opportunity of achieving pregnancy is limited; for these individuals, assisted reproductive techniques should be considered undertaking careful and adequate preventive and therapeutic measures, for example, in case of women positivity, the use of human monoclonal antibody against SARS-CoV-2 isolated from memory B cells of an individual infected with SARS-CoV in 2003 [24] or vaccine when it will be available.

Authors' contributions LZ performed data acquisition and drafted the manuscript. LP contributed to data acquisition and interpretation and drafted and critically revised the manuscript. GZ contributed to data interpretation and drafted the manuscript. GR contributed to data interpretation and critically revised the manuscript. SC conceived the study; contributed to data acquisition, analysis, and interpretation; and critically revised the manuscript.
Funding information The project is supported by the Interreg ItaliaSlovenia, ISE-EMH 07/2019, RC03/20 BioHub research project and 5mille15D1 from IRCCS Burlo Garofolo and Italian Ministry of Health.

\section{Compliance with ethical standards}

Conflict of interest The authors declare that they have no conflict of interest.

\section{References}

1. Tu Y-F, Chien C-S, Yarmishyn AA, Lin Y-Y, Luo Y-H, Lin Y-T, et al. A review of SARS-CoV-2 and the ongoing clinical trials. Int J Mol Sci. 2020;21:2657.

2. Wang S, Zhou X, Zhang T, Wang Z. The need for urogenital tract monitoring in COVID-19. Nat Rev Urol. 2020;17:314-5.

3. Nicin L, Abplanalp WT, Mellentin H, Kattih B, Tombor L, John D, et al. Cell type-specific expression of the putative SARS-CoV-2 receptor ACE2 in human hearts. Eur Heart J. 2020;41:1804-6.

4. Jing Y, Run-Qian L, Hao-Ran W, Hao-Ran C, Ya-Bin L, Yang G, et al. Potential influence of COVID-19/ACE2 on the female reproductive system. Mol Hum Reprod. 2020;26:367-73.

5. Younis JS, Abassi Z, Skorecki K. Is there an impact of the COVID19 pandemic on male fertility? The ACE2 connection. Am J Physiol Endocrinol Metab. 2020;318:E878-80.

6. Hoffmann M, Kleine-Weber H, Schroeder S, Krüger N, Herrler T, Erichsen S, et al. SARS-CoV-2 cell entry depends on ACE2 and TMPRSS 2 and is blocked by a clinically proven protease inhibitor. Cell. 2020;181:1-10.

7. Ou X, Liu Y, Lei X, Li P, Mi D, Ren L, et al. Characterization of spike glycoprotein of SARS-CoV-2 on virus entry and its immune cross-reactivity with SARS-CoV. Nat Commun. 2020;11:1620.

8. Pan P-P, Zhan Q-T, Le F, Zheng Y-M, Jin F. Angiotensinconverting enzymes play a dominant role in fertility. Int J Mol Sci. 2013;14:21071-86.

9. Wang Z, Xu X. scRNA-seq profiling of human testes reveals the presence of the ACE2 receptor, a target for SARS-CoV-2 infection in spermatogonia, Leydig and Sertoli Cells. Cells. 2020;9:920.

10. Zhang J, Wu Y, Wang R, Lu K, Tu M, Guo H, et al. Bioinformatic analysis reveals that the reproductive system is potentially at risk from SARS-CoV-2. other; 2020.

11. Verma S, Saksena S, Sadri-Ardekani H. ACE2 receptor expression in testes: Implications in COVID-19 Pathogenesis. Biol Reprod. 2020.

12. Liu X, Chen Y, Tang W, Zhang L, Chen W, Yan Z, et al. Single-cell transcriptome analysis of the novel coronavirus (SARS-CoV-2) associated gene ACE2 expression in normal and non-obstructive azoospermia (NOA) human male testes. Sci China Life Sci. 2020.

13. Stanley KE, Thomas E, Leaver M, Wells D. Coronavirus disease19 and fertility: viral host entry protein expression in male and female reproductive tissues. Fertil Steril. 2020;114:33-43.

14. Paoli D, Pallotti F, Turriziani O, Mazzuti L, Antonelli G, Lenzi A, et al. SARS-CoV-2 presence in seminal fluid: myth or reality. Andrology. 2020.

15. Pan F, Xiao X, Guo J, Song Y, Li H, Patel DP, et al. No evidence of severe acute respiratory syndrome-coronavirus 2 in semen of males recovering from coronavirus disease 2019. Fertil Steril. 2020;113: 1135-9.

16. Paoli D, Pallotti F, Colangelo S, Basilico F, Mazzuti L, Turriziani $\mathrm{O}$, et al. Study of SARS-CoV-2 in semen and urine samples of a volunteer with positive naso-pharyngeal swab. J Endocrinol Investig. 2020. 
17. Li D, Jin M, Bao P, Zhao W, Zhang S. Clinical characteristics and results of semen tests among men with coronavirus disease 2019. JAMA Netw Open. 2020;3:e208292.

18. Xu J, Qi L, Chi X, Yang J, Wei X, Gong E, et al. Orchitis: a complication of severe acute respiratory syndrome (SARS). Biol Reprod. 2006;74:410-6.

19. Henarejos-Castillo I, Sebastian-Leon P, Devesa-Peiro A, Pellicer A, Diaz-Gimeno P. SARS-CoV-2 infection risk assessment in the endometrium: viral infection-related gene expression across the menstrual cycle. Fertil Steril. 2020;114:223-32.

20. De Bernardo G, Giordano M, Zollo G, Chiatto F, Sordino D, De Santis R, et al. The clinical course of SARS-CoV-2 positive neonates. J Perinatol. 2020.

21. Dong L, Tian J, He S, Zhu C, Wang J, Liu C, et al. Possible vertical transmission of SARS-CoV-2 from an infected mother to her newborn. JAMA. 2020.
22. Zeng H, Xu C, Fan J, Tang Y, Deng Q, Zhang W, et al. Antibodies in infants born to mothers with COVID-19 pneumonia. JAMA. 2020.

23. Wang W, Xu Y, Gao R, Lu R, Han K, Wu G, et al. Detection of SARS-CoV-2 in different types of clinical specimens. JAMA. 2020;323:1843-4.

24. Pinto D, Park Y-J, Beltramello M, Walls AC, Tortorici MA, Bianchi S, et al. Cross-neutralization of SARS-CoV-2 by a human monoclonal SARS-CoV antibody. Nature. 2020;583:290-5.

Publisher's note Springer Nature remains neutral with regard to jurisdictional claims in published maps and institutional affiliations. 